\title{
Análisis de los factores predictivos de conversión en cirugía laparoscópica colorrectal*
}

\author{
Drs. GONZALO INOSTROZA L. ${ }^{1}$, GONZALO URREJOLA S. ${ }^{1}$, GINO CASELLI M. ${ }^{1}$, \\ MARÍA E. MOLINA P. ${ }^{1}$, FELIPE BELLOLIO R. ${ }^{1}$, ÁLVARO ZÚNIIGA D. ${ }^{1}$, \\ E.U. XIMENA MENESES C. ${ }^{1}$, Int. MÓNICA MARTÍNEZ M. ${ }^{2}$, Dr. GEORGE PINEDO M. ${ }^{1}$ \\ 1 Unidad de Cirugía Colorrectal, Departamento de Cirugía Digestiva, Facultad de Medicina, Pontificia Universidad \\ Católica de Chile. \\ 2 Interna, Facultad de Medicina, Pontificia Universidad Católica de Chile. \\ Santiago, Chile.
}

\begin{abstract}
Analysis of predictive factors for conversion to open surgery in laparoscopic colorectal surgery

Background: Conversion to open surgery of laparoscopic procedures is not in essence a complication, but invalidates the benefits of laparoscopy. Aim: To identify the predictive factors for conversion in laparoscopic colorectal surgery. Material and Methods: Revision of medical records of all patients with colorectal disease operated using a laparoscopic approach, from 1998 to 2010. Gender, age, American Society of Anesthesiologists (ASA) score, body mass index (BMI), previous abdominal surgery, elective/urgency procedure, benign/malignant disease, type of resection and surgeon experience were recorded. A logistic regression model was done to determine which variables were predictive for conversion to open surgery. Results: The medical records of 582 patients aged $57 \pm 17$ years $(45 \%$ men) were analyzed. The rate of conversion to open surgery was $7.1 \%$. The logistic regression model selected as predictors of conversion a BMI over $25 \mathrm{~kg} / \mathrm{m}^{2}$ (odds ratio (OR) 4.9, 95\% confidence intervals (CI) 2.4 to 9.9), cancer surgery (OR 2.1, 95\% CI 1.1 to 4.3 ) and male sex (OR 2.30, 95\% CI 1.14 to 4.65). The receiver operating curve (ROC) of the model had an are under the curve of 0.766 with $95 \%$ CI of 0.69 to 0.84 ). Conclusions: A BMI over $25 \mathrm{~kg} / \mathrm{m}^{2}$, male sex and the resection of a malignant tumor were predictive factors for conversion to open surgery.
\end{abstract}

Key words: Laparoscopic colorectal surgery, conversion, predictive factors.

\section{Resumen}

Objetivo: Identificar los factores de riesgo para la conversión en la cirugía laparoscópica colorrectal. Material y Método: Se revisó la base de datos prospectiva de cirugía laparoscópica colorrectal, desde 1998 a 2010. Se analizaron las variables: sexo, edad, ASA, IMC, presencia de cirugía abdominal previa, procedimiento electivo/urgencia, patología benigna/maligna, tipo de resección y experiencia del cirujano. Se realizó un análisis uni y multivariado. Para determinar las variables predictivas de conversión, la totalidad de estas

\footnotetext{
* Recibido el 29 de octubre de 2010 y aceptado para publicación el 30 de diciembre de 2010.

Correspondencia: Dr. Gonzalo Inostroza L.

Alameda 351, Dpto. 415, Santiago, Chile.

gonzalo.inostroza.levy@gmail.com
} 
fueron incluidas en un modelo de regresión logística. Resultados: De un total de 621 pacientes consecutivos, la serie se compuso de 582 pacientes (hombres: 45\%, edad promedio: 56,3 años) Tasa de conversión 7,1\%. El modelo de regresión logística seleccionó tres variables como predictivas de conversión: IMC $>25 \mathrm{~kg} / \mathrm{m}^{2}$ (OR 4,88; IC95\% 2,40-9,92), cirugía por cáncer (OR 2,12; IC95\% 1,11-4,29) y sexo masculino (OR 2,30; IC95\% 1,14-4,65). No fueron predictivas de conversión: edad, comorbilidades, experiencia del cirujano, tipo de procedimiento, ni cirugía previa. La calibración del modelo fue satisfactoria, al igual que su capacidad de discriminación $(\mathrm{ABC} \mathrm{ROC}=0,766)$. Conclusiones: En este estudio el IMC sobre $25 \mathrm{~kg} / \mathrm{m}^{2}$, el sexo masculino y las resecciones por cáncer son factores predictivos independientes de conversión. Este modelo predictivo mostró una calibración satisfactoria, asociada a una capacidad de discriminación acertada para el evento en estudio.

Palabras clave: Cirugía laparoscópica colorrectal, conversión, factores predictivos.

\section{Introducción}

La primera colecistectomía laparóscopica se realizó a mediados de los 80 y desde su introducción se ha convertido en el gold standard para el tratamiento de la litiasis biliar. Los excelentes resultados obtenidos con este abordaje se han intentado replicar en diversas áreas de la cirugía ${ }^{1}$. En el año 1991 se realizó el primer procedimiento colorrectal laparoscópico ${ }^{2}$ y desde entonces se ha aplicado para la patología benigna y para el cáncer colorrectal. En los últimos años se han demostrado los beneficios a corto plazo del abordaje mínimamente invasivo con resultados oncológicos que son comparables a los obtenidos en cirugía convencional ${ }^{3}$, pudiendo considerarse actualmente como vía de elección para la patología colorrectal $^{3-5}$. En un número no despreciable de ocasiones el cirujano debe realizar la conversión a cirugía tradicional abierta ${ }^{5}$. A pesar del avance en la técnica y el perfeccionamiento de los crujanos, las distintas series internacionales muestran tasas de conversión que van desde un 2 a un $40 \%{ }^{5-12}$.

Es aceptado que la conversión no es en sí misma una complicación ${ }^{11}$, sino una herramienta para completar en forma segura un procedimiento, pero su impacto en los resultados está en discusión ${ }^{7}$. La conversión trae consigo la pérdida de los beneficios de la cirugía mínimamente invasiva y a su vez adiciona los costos del procedimiento ${ }^{7}$. Por lo anterior, se ha tratado de definir a priori qué pacientes tienen un mayor riesgo de conversión y generar modelos de predicción de este suceso ${ }^{5,7,8,10-12}$.

El objetivo de este trabajo es identificar los factores de riesgo para la conversión en la cirugía laparoscópica colorrectal en un centro de referencia especializado.

\section{Material y Método}

Con el objetivo de determinar los factores de riesgo para conversión de cirugía laparoscópica colorrectal, se revisó la base de datos prospectiva de los pacientes sometidos a cirugía colorrectal laparoscópica en el Hospital Clínico de la Pontificia Universidad Católica de Chile desde enero de 1998 a mayo de 2010. La serie está compuesta por 621 pacientes operados consecutivamente, excluyéndose aquellos en que se desconocía, por falta de registro, la variable conversión. Se obtuvo un total de 582 pacientes para el estudio. Se consideró las variables demográficas: sexo, edad, Score de ASA, índice de masa Corporal (IMC), la presencia de cirugía abdominal previa (si/no), tipo de patología (benigna o maligna), cirugía de urgencia (si/no), Se clasificó los pacientes según el número de procedimientos laparoscópicos llevados a cabo previamente por el cirujano a cargo de su intervención. Así los cirujanos fueron divididos en aquellos con 100 o más procedimientos y menos de 100 .

Se dividió al grupo en tres partes iguales según la posición correlativa que ocuparon en la serie, conformándose 3 grupos de 183 pacientes.

Definición de conversión: Laparotomía no programada en el procedimiento quirúrgico, mediante la cual el cirujano fuese capaz de introducir su mano para completar la intervención ${ }^{13}$.

\section{Análisis estadístico}

Las variables fueron descritas en términos de número y porcentaje, y su asociación evaluada mediante la prueba de $\chi^{2}$. Para determinar las variables predictivas de conversión, la totalidad de las variables fueron incluidas en un modelo de regresión logística (modalidad Wald), obteniéndose odds ratio ajustados e intervalos de confianza de $95 \%$. La calibración del modelo fue evaluada mediante la prueba de Hosmer y Lemeshow y su discriminación mediante c-index (área bajo la curva ROC). Se consideró como estadísticamente significativo todo valor $p$ menor a 0,05 . Los cálculos fueron realizados utilizando el software SPSS versión 18.0 . 


\section{Resultados}

Quinientos ochenta y dos pacientes conformaron el grupo de estudio. Se encontraron 261 pacientes de sexo masculino (44,8\%). La edad promedio $56,6 \pm$ 16,6 años y el IMC promedio fue $24,1 \pm 3,2$. La tasa de conversión global fue de 7,1\%.

En el análisis univariado cuatro variables fueron identificadas como predictivas de conversión. La tasa de conversión fue significativamente mayor en los pacientes de sexo masculino $(10,1 \%$ vs $4,1 \%)$, en las resecciones por cáncer ( $10,4 \%$ vs $4,8 \%)$, en aquellos pacientes con IMC mayor o igual de 25 $\mathrm{kg} / \mathrm{m}^{2}(14,6 \%$ vs $3,1 \%)$ y en aquellos sometidos a intervenciones de urgencia $(20,8 \%$ vs $6,5 \%)$. Las demás variables no presentaron diferencias entre ambos grupos. Los resultados de la serie y análisis univariado se muestran en la Tabla 1.

El modelo de regresión logística (análisis multivariado) seleccionó tres variables como predictivas de conversión, IMC $>25 \mathrm{~kg} / \mathrm{m}^{2}$ (OR 6,04; IC95\% 2,85-12,92), cirugía por cáncer (OR 2,01; IC95\%

Tabla 1. Análisis univariado de las características de la serie

\begin{tabular}{|c|c|c|c|}
\hline \multirow[t]{2}{*}{ Variable } & \multicolumn{2}{|c|}{ Conversión, n (\%) } & \multirow[t]{2}{*}{ Valor $\mathbf{p}$} \\
\hline & $\begin{array}{l}\text { Positivo } \\
(\mathrm{n}=41)\end{array}$ & $\begin{array}{l}\text { Negativo } \\
(\mathrm{n}=581)\end{array}$ & \\
\hline \multicolumn{4}{|l|}{ Edad (años) } \\
\hline Menor a 65 & $27(6,8)$ & $373(93,3)^{*}$ & 0,617 \\
\hline $65+$ & $14(7,9)$ & $163(92,1)^{*}$ & \\
\hline \multicolumn{4}{|l|}{ Sexo } \\
\hline Femenino & $13(4,1)$ & $307(95,9)$ & 0,002 \\
\hline Masculino & $28(10,7)$ & $234(89,3)$ & \\
\hline \multicolumn{4}{|l|}{ ASA } \\
\hline I & $13(4,9)$ & $254(95,1)$ & 0,059 \\
\hline II y III & $28 \quad(8,9)$ & $287(91,1)$ & \\
\hline \multicolumn{4}{|l|}{ Cirugía previa } \\
\hline Sí & $22(8,2)$ & $247(91,8)$ & 0,322 \\
\hline No & $19(6,1)$ & $294(93,9)$ & \\
\hline \multicolumn{4}{|l|}{ IMC $\left(\mathrm{kg} / \mathrm{m}^{2}\right)$} \\
\hline Menor a 25 & $12(3,1)$ & $371(96,9)$ & $<0,001$ \\
\hline $25+$ & $29(14,6)$ & $170(85,4)$ & \\
\hline \multicolumn{4}{|l|}{ Cirugía rectal } \\
\hline Sí & $6(5,3)$ & $108(94,7)$ & 0,532 \\
\hline No & $35(7,5)$ & $433(92,5)$ & \\
\hline \multicolumn{4}{|c|}{ Experiencia del cirujano } \\
\hline Menos de 100 & $7(7,2)$ & $90(92,8)$ & 1,000 \\
\hline $100+$ & $34(7,0)$ & $451(93,0)$ & \\
\hline \multicolumn{4}{|c|}{ Cirugía por cáncer } \\
\hline Sí & $24(10,4)$ & $206(89,6)$ & 0,010 \\
\hline No & $17(4,8)$ & $335(95,2)$ & \\
\hline \multicolumn{4}{|c|}{ Cirugía de Urgencia } \\
\hline Sí & $5(20,8)$ & $19(79,2)$ & 0,022 \\
\hline No & $36(6,5)$ & $522(93,5)$ & \\
\hline \multicolumn{4}{|c|}{ Período de cirugía } \\
\hline 0 & $16(8,3)$ & $177(91,7)$ & 0,440 \\
\hline 1 & $10(5,2)$ & $184(94,8)$ & \\
\hline 2 & $15(7,7)$ & $180(92,3)$ & \\
\hline
\end{tabular}


ANÁLISIS DE LOS FACTORES PREDICTIVOS DE CONVERSIÓN EN CIRUGÍA LAPAROSCÓPICA COLORRECTAL

Tabla 2. Regresión Logística de factores de riesgo de conversión en cirugía laparoscópica colorrectal

\begin{tabular}{lrrrrrrrr}
\hline & B & E.T. & Wald & gl & Sig. & OR & \multicolumn{2}{c}{ I.C. 95\% para EXP(B) } \\
& & & & & & & Inferior & Superior \\
\hline Sexo masculino &, 889 &, 369 & 5,795 & 1 &, 016 & 2,433 & 1,180 & 5,017 \\
Cirugía por cáncer &, 742 &, 351 & 4,461 & 1 &, 035 & 2,100 & 1,055 & 4,180 \\
IMC $\geq 25$ & 1,798 &, 383 & 22,059 & 1 &, 000 & 6,039 & 2,851 & 12,789 \\
Constante & $-4,428$ &, 444 & 99,293 & 1 &, 000 &, 012 & & \\
\hline
\end{tabular}

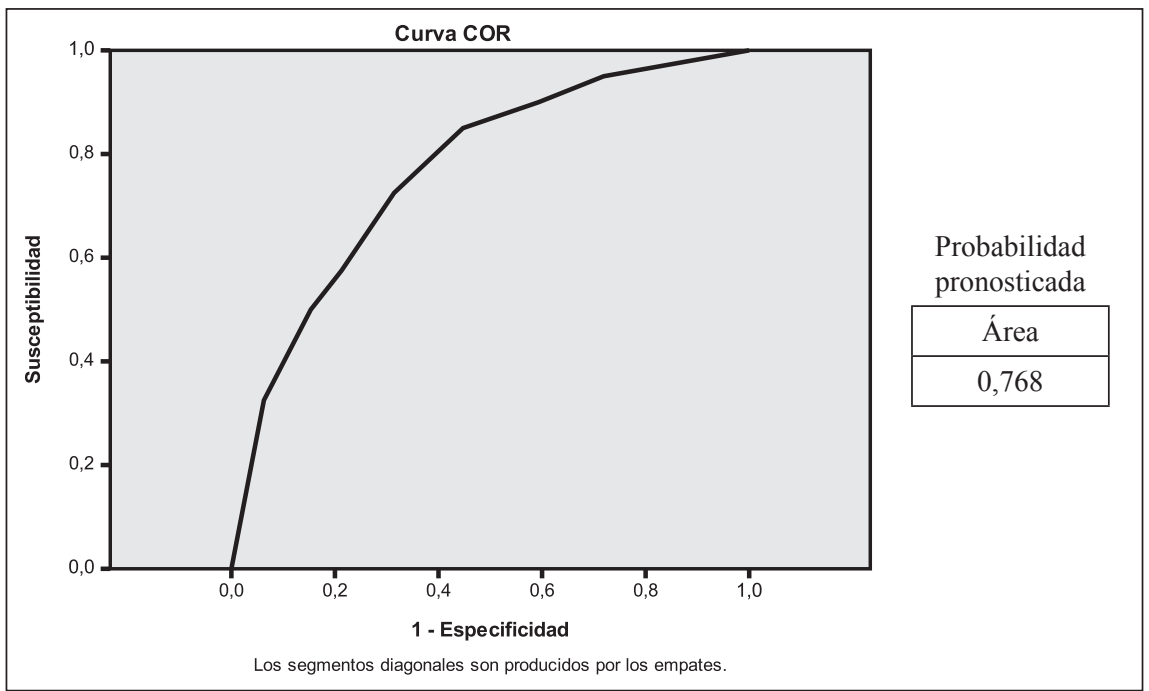

Figura 1. Curva ROC modelo.
$1,05-4,18)$ y sexo masculino (OR 2,43; IC95\% 1,18 $5,02)$, quedando fuera la variable cirugía de urgencia (Tabla 2).

La calibración del modelo fue satisfactoria (valor p prueba de Hosmer y Lemeshow $=0,872$ ), al igual que su capacidad de discriminación (área bajo la curva $\mathrm{ROC}=0,768$; IC95\% 0,691-0,841) (Figura 1).

\section{Discusión}

En este estudio, el análisis multivariable entregó tres factores predictivos de conversión. A saber: el IMC sobre 25, el sexo masculino y la cirugía por patología maligna, todos con significación estadística.

La obesidad y el sobrepeso se han considerado desde el inicio de la cirugía laparoscópica como factores que aumentan la dificultad técnica del procedimiento. En este estudio un IMC sobre 25 (sobrepeso) se consideró un factor de riesgo independiente para conversión, lo que concuerda con lo publicado. Al respecto, el grupo de Pikarski ${ }^{14}$, publicó un riesgo 2,2 veces mayor de conversión en pacientes con IMC sobre 30, mientras que Tekkis ${ }^{11}$, pone como valor de corte $28,5 \mathrm{~kg} / \mathrm{m}^{2}$. Algunos estudios han considerado el IMC elevado como factor de exclusión, como es el caso del Dutch Trial Colorectal Resection ${ }^{15}$. Rotholtz ${ }^{16}$, publicó en sus resultados, que a pesar de ser más complejo el escenario en los pacientes obesos, estos no presentaron mayores tasas de conversión que el resto de la población en estudio.

A pesar de esto, es importante señalar que la cirugía laparoscópica de colon es segura de realizar en pacientes con sobrepeso y obesidad, por lo tanto, no se pueden negar los potenciales beneficios de este abordaje a este importante grupo de pacientes, basados única y exclusivamente en el IMC.

Las comorbilidades se han asociado generalmente a mayor tasa de complicación y mortalidad en la cirugía colorrectal, pero su relación con la conversión de la cirugía laparoscópica también ha sido analizada, existiendo grupos que la señalan como predictivas, o quienes incluso las excluyen de 
los análisis por considerar que no existe evidencia para considerarlo un factor de riesgo. En esta serie al analizar el Score de ASA como factor de riesgo de conversión, este rozó la significación estadística en el análisis univariado, pero no fue determinante en el modelo predictivo multivariado.

Con respecto al sexo de los pacientes, el grupo de Thorpe ${ }^{7}$, determinó que la anatomía masculina representa una dificultad en la cirugía laparoscópica. El menor espacio en la pelvis puede dificultar la movilización de un tumor de sigmoides o de recto alto. Schlachta ${ }^{8}$, también demostró que el sexo masculino resultó un factor de riesgo de conversión $(13,6 \%$ vs $4,4 \% ; p=0,0037)$, pero esta relación estaba mediada por un mayor sobrepeso de los pacientes masculinos (análisis multivariable). En la presente serie se apoyan estos resultados siendo una posible explicación la relación existente entre el sexo masculino y mayor grasa visceral, la cual podría evaluarse mediante algunos estudios imagenológicos.

En los estudios de Tekkis ${ }^{11}$ y de Pandya ${ }^{17}$, el cáncer no fue un factor de riesgo, a diferencia de lo comunicado por Thorpe ${ }^{7}$, para quien los tumores localmente avanzados fueron factores condicionantes de conversión, al igual que los resultados de este estudio. La fijación del tumor a estructuras adyacentes, la menor visibilidad por falta de espacio o la dificultad para manipular la lesión hacen del cáncer un condicionante para conversión. En nuestro estudio, el factor neoplásico, llevó probablemente al cirujano a la cautela frente a la sospecha de estar localmente avanzado y muchas veces, especialmente en la curva inicial, se decidió la conversión frente a la duda.

Similar a lo publicado por Bennet ${ }^{18}$, en esta experiencia no hubo diferencias entre cirujanos con mayor número de procedimientos laparoscópicos y aquellos con menos procedimientos. Los resultados en esta experiencia podrían explicarse por diversos motivos. Primero, debe considerarse que en esta serie más del $60 \%$ del total de los procedimientos es realizado por 3 cirujanos, por lo que son ellos quienes más realizan conversiones. En segundo lugar, siempre uno de los involucrados es de aquellos con mayor experiencia. De esta forma, muchas veces un residente es el cirujano principal, pero es guiado por un miembro experimentado del equipo. Marusch et $\mathrm{al}^{19}$, en un estudio multicéntrico con más de 1.000 pacientes, cirujanos con más de 100 procedimientos se embarcan en procedimientos más complejos, lo que produce una curva bimodal en el gráfico de conversión. Así mismo, tampoco, se encontró diferencias entre los primeros 200 casos y el resto de la serie. Schwandner et $\mathrm{al}^{20}$, señaló que factores similares a la experiencia individual son aplicables a un equipo quirúrgico.

En esta serie la tasa de conversión en pacientes con cirugía previa fue de $8,2 \%$ en 269 , lo que no fue un factor predictivo de conversión. La experiencia obtenida en otros procedimientos laparoscópicos de menor complejidad, ha permitido que en la actualidad las intervenciones previas no se consideren una contraindicación para el abordaje laparoscópico en cirugía de colon.

En este estudio la tasa de conversión de aquellos pacientes intervenidos de urgencia fue de 20,8\% siendo ésta mucho mayor que en los pacientes operados electivamente $(6,5 \%)$. A pesar de esta diferencia y de ser estadísticamente significativo en el análisis univariado, en el modelo de regresión esta variable no resultó ser un factor predictivo de conversión. Los eventos intraoperatorios propios de la cirugía de urgencia (abscesos y perforaciones) son factores de riesgo de conversión en el estudio de Tekkis $^{11}$. En el metanalisis de Gervaz ${ }^{5}$, que incluye 28 estudios, con más de 3.232 pacientes, también se cita a la cirugía de urgencia como factor de riesgo de conversión, en especial aquella relacionada con diverticulitis aguda. Algunos grupos han propuesto que la conversión en cirugía de urgencia decrecerá en forma paulatina a medida que aumente la experiencia en este tipo de procedimientos.

\section{Conclusión}

En esta serie, el sexo masculino, el sobrepeso (IMC sobre $25 \mathrm{~kg} / \mathrm{m}^{2}$ ) y las resecciones por cáncer resultaron ser factores predictivos de conversión. Debe destacarse que ninguna de estas condiciones es considerada una contraindicación para el abordaje laparoscópico, se debe enfatizar en la correcta selección de los pacientes para las distintas alternativas de abordaje.

\section{Referencias}

1. Rico Selas P, Calle Santiuste A. Laparoscopic cholecystectomy and outpatient surgery. Rev Esp Enferm Dig. 2004;96:435-41.

2. Jacobs M, Verdeja JC, Goldstein HS. Minimally invasive colon resection (laparoscopic colectomy). Surg Laparosc Endosc. 1991;1:144-50.

3. Kuhry E, Schwenk W, Gaupset R, Romild U, Bonjer J. Long-term outcome of laparoscopic surgery for colorectal cancer: a Cochrane systematic review of randomised controlled trials. Cancer Treat Rev. 2008;34:498-504.

4. Schwenk W, Haase O, Neudecker J, Muller JM. Short term benefits for laparoscopic colorectal resection. Cochrane Database Syst Rev. 2005:CD003145.

5. Gervaz P, Pikarsky A, Utech M, Secic M, Efron J, Belin $\mathrm{B}$, et al. Converted laparoscopic colorectal surgery. Surg Endosc. 2001;15:827-32. 
6. Avital S, Hermon H, Greenberg R, Karin E, Skornick Y. Learning curve in laparoscopic colorectal surgery: our first 100 patients. Isr Med Assoc J. 2006;8:683-6.

7. Thorpe H, Jayne DG, Guillou PJ, Quirke P, Copeland J, Brown JM. Patient factors influencing conversion from laparoscopically assisted to open surgery for colorectal cancer. Br J Surg. 2008 ;95:199-205.

8. Schlachta CM, Mamazza J, Seshadri PA, Cadeddu MO, Poulin EC. Predicting conversion to open surgery in laparoscopic colorectal resections. A simple clinical model. Surg Endosc. 2000;14:1114-7.

9. Degiuli M, Mineccia M, Bertone A, Arrigoni A, Pennazio $M$, Spandre $M$, et al. Outcome of laparoscopic colorectal resection. Surg Endosc. 2004;18:427-32.

10. Tan PY, Stephens JH, Rieger NA, Hewett PJ. Laparoscopically assisted colectomy: a study of risk factors and predictors of open conversion. Surg Endosc. 2008; 22:1708-14.

11. Tekkis PP, Senagore AJ, Delaney CP. Conversion rates in laparoscopic colorectal surgery: a predictive model with, 1253 patients. Surg Endosc. 2005;19:47-54.

12. Murillo ZA, Posada T JA. Factores predictivos de conversión en la sigmoidectomia laparoscópica para el tratamiento de le enfermedad diverticular. Rev Chil Cir. 2009;61:443-7.

13. Shawki S, Bashankaev B, Denoya P, Seo C, Weiss EG, Wexner SD. What is the definition of "conversion" in laparoscopic colorectal surgery? Surg Endosc. 2009;23:
2321-6.

14. Pikarsky AJ, Saida Y, Yamaguchi T, Martínez S, Chen W, Weiss EG, et al. Is obesity a high-risk factor for laparoscopic colorectal surgery? Surg Endosc. 2002;16:8558.

15. Wiggers T, van de Velde CJ. The circumferential margin in rectal cancer. Recommendations based on the Dutch Total Mesorectal Excision Study. Eur J Cancer. 2002; 38:973-6.

16. Rotholtz NA, Laporte M, Zanoni G, Bun ME, Aued L, Lencinas S, et al. Predictive factors for conversion in laparoscopic colorectal surgery. Tech Coloproctol. 2008;12:27-31.

17. Pandya S, Murray JJ, Coller JA, Rusin LC. Laparoscopic colectomy: indications for conversion to laparotomy. Arch Surg. 1999;134:471-5.

18. Bennett CL, Stryker SJ, Ferreira MR, Adams J, Beart RW Jr. The learning curve for laparoscopic colorectal surgery. Preliminary results from a prospective analysis of 1194 laparoscopic-assisted colectomies. Arch Surg. 1997;132:41-4; discussion 5.

19. Marusch F, Gastinger I, Schneider C, Scheidbach H, Konradt J, Bruch HP, et al. Experience as a factor influencing the indications for laparoscopic colorectal surgery and the results. Surg Endosc. 2001;15:116-20.

20. Schwandner O, Schiedeck TH, Bruch H. The role of conversion in laparoscopic colorectal surgery: Do predictive factors exist? Surg Endosc. 1999;13:151-6. 